\title{
Effects of the downregulation of SnoN expression on HepG2 cell proliferation and apoptosis
}

\author{
WENQI WANG ${ }^{1}$, CHANGHONG LIU $^{1}$, YIGUO WANG ${ }^{1}$ and LILI CAO ${ }^{2}$ \\ ${ }^{1}$ Department of Gastroenterology and ${ }^{2}$ General Laboratory, Qianfoshan Hospital Affiliated to Shandong University, \\ Jinan, Shandong 250014, P.R. China
}

Received November 23, 2012; Accepted January 31, 2013

DOI: $10.3892 / \mathrm{mmr} .2013 .1340$

\begin{abstract}
Ski-novel protein (SnoN) is a proto-oncogene that belongs to the Ski protein family and is involved in regulating processes such as cell proliferation and apoptosis. To investigate the role of SnoN in the proliferation and apoptosis of HepG 2 cells, we downregulated its expression by the use of small interfering RNA (siRNA). Three fragments predicted to have RNAi capacity were designed and synthesized as the target siRNAs (siRNA-A, -B and -C). Following transfection, inhibition efficiency was detected by reverse transcription PCR (RT-PCR) and western blot analysis. The siRNA with the optimal inhibition efficiency was used for the cell proliferation and apoptosis analysis. Cell proliferation was analyzed by the Cell Counting Kit-8 (CCK-8) and cell apoptosis was investigated by flow cytometry. In our study, all three siRNAs efficiently inhibited SnoN expression, and siRNA-C demonstrated the optimal inhibition efficiency. We found that following downregulation of SnoN expression, HepG2 cell proliferation was significantly inhibited $(\mathrm{P}<0.05)$, while HepG2 cell apoptosis was significantly increased $(\mathrm{P}<0.05)$. SnoN-specific siRNA is capable of effectively inhibiting the expression of SnoN in human HepG2 cells, and the downregulation of SnoN expression induces growth inhibition and apoptosis.
\end{abstract}

\section{Introduction}

Ski-novel protein $(\mathrm{SnoN})$ is a proto-oncogene that belongs to the Ski protein family and has diverse biological activities as a nuclear oncoprotein (1). It is important in regulating cell proliferation, differentiation, senescence and apoptosis $(2,3)$. Studies have demonstrated that $\mathrm{SnoN}$ is a crucial negative regulator of the TGF $\beta /$ Smad signaling pathway (4) and an activator of P53 $(5,6)$. The human proto-oncogene, the SnoN

Correspondence to: Dr Wenqi Wang, Department of Gastroenterology, Qianfoshan Hospital Affiliated to Shandong University, 16766 Jingshi Road, Jinan, Shandong 250014, P.R. China E-mail: wangwq62@163.com

Key words: small interfering RNA, SnoN, liver neoplasms, apoptosis gene, is located on the $3 q 26$ chromosome, which encodes SnoN (a 64 amino acid) protein (1). In normal tissues and cells, SnoN protein is predominantly expressed in the cytoplasm; however, in the majority of tumor tissues and cells, SnoN protein is mainly expressed in the nucleus (7). SnoN acts as a co-repressor of Smad proteins to negatively regulate the TGF- $\beta /$ Smad signaling pathway (8). A previous study demonstrated that SnoN interacts with Smad4 and disrupts functional heteromeric Smad complexes, which blocks the translocation of the Smad complexes into the nucleus and then represses their transactivation activities (9). Thus far, the role of SnoN in tumor progression remains controversial. Consistent with its role as an oncoprotein, SnoN expression is elevated in numerous human tumor tissues and cell lines, while in normal cells its expression level is relatively low or undetectable (10). One study demonstrated that SnoN exerted oncogenic effects in human colorectal carcinogenesis. Additionally, elevation in SnoN expression was correlated with the activation of the $\beta$-catenin pathway in those colorectal carcinomas (11). However, SnoN also possess anti-oncogenic activity, as demonstrated by Shinagawa et al who found that a loss of SnoN expression in mice increased their susceptibility to chemical carcinogen-induced tumorigenesis (12). Furthermore, SnoN was able to stabilize P53 by interacting with other factors. Another recent study provided the first in vivo evidence of a pro-oncogenic role for $\mathrm{SnoN}$ in mammalian tumorigenesis. Transgenic mice that could express elevated levels of SnoN were generated, and it was found that elevated SnoN expression was insufficient to induce tumorigenesis in the mice (13). However, elevated SnoN expression accelerated the progression of the tumors. Overall, SnoN expression is closely correlated with tumorigenesis. However, the roles and mechanisms of SnoN in tumorigenesis are significantly more complex. Therefore, these require further investigation. Numerous studies of the roles and mechanisms of SnoN have been conducted in colon, lung and esophageal cancer $(11,14,15)$. The role and mechanism of $\mathrm{SnoN}$ in liver cancer is not yet clear. However, the role of SnoN in hepatocarcinoma development has rarely been studied.

RNA interference (RNAi) is a powerful research tool that has selective and robust effects on gene expression. It is capable of interfering with the expression of specific genes containing complementary double-stranded RNA (dsRNA) nucleotide sequences. Small interfering RNA (siRNA) is one of two types of RNAi. It is a short, double-stranded RNA 
catalyzed by the Dicer enzyme (a member of the RNAase III family that specifically catalyzes double-stranded RNAs). These small RNAs are able to bind to other specific complementary messenger RNA (mRNA) molecules and block their activities (16). siRNA technology is widely used in cancer research. In this study, we blocked SnoN gene expression by the use of siRNA silencing in HepG2 cells, and investigated its role in hepatocarcinoma development.

\section{Materials and methods}

Reagents and antibodies. DMEM and $0.25 \%$ trypsin were purchased from Hyclone Laboratories Inc. (Logan, UT, USA); fetal bovine serum was purchased from Zhejiang Tianhang Biotechnology Co., Ltd. (China) and Opti-MEM medium was purchased from Gibco (Carlsbad, CA, USA). Lipofectamine $^{\mathrm{TM}}$ 2000, TRIzol reagent and annexin V/PI apoptosis detection kit were purchased from Invitrogen Life Technologies (Carlsbad, CA, USA). Reverse transcription RNA PCR kit (AMV) version 3.0 was purchased from Takara Biotechnology (Dalian) Co., Ltd. (China). EasyTaq mix and DNA markers were purchased from TransGen Biotech, Co., Ltd. (Beijing, China). Cell Counting Kit-8 (CCK-8) was purchased from Dojindo Laboratories (Kumamoto, Japan). Anti-SnoN polyclonal antibody was purchased from Abcam (New Territories, Hong Kong) and anti- $\beta$-actin monoclonal antibody was obtained from Wuhan Boster Bio-Engineering Limited Company (Hubei, China).

Cell lines and cultures. The HepG2 human hepatocellular liver carcinoma cell line was obtained from the Key Laboratory of General Surgery, Shandong, China. Cells were cultured in DMEM medium (Hyclone Laboratories Inc.) supplemented with $10 \%$ fetal bovine serum (Zhejiang Tianhang Biotechnology Co., Ltd.) and $100 \mathrm{U} / \mathrm{ml}$ penicillin/streptomycin (Gibco), and maintained in a humidified incubator $\left(5 \% \mathrm{CO}_{2}\right)$ at $37^{\circ} \mathrm{C}$.

Design and synthesis of siRNAs. The siRNAs used in this study were designed based on the SnoN gene sequence in GeneBank (NM_005414). According to the design rules for RNAi (17), three fragments that were predicted to have RNAi capacity were used as the target siRNA (siRNA-A, -B and -C). A negative control siRNA, which does not target any known gene, was also designed (Table I). All the siRNAs were purchased from Shanghai Genepharma Co., Ltd (China).

siRNA transfection. siRNA transfection was performed using Lipofectamine 2000 (Invitrogen Life Technologies) according to the manufacturer's instructions. Briefly, one day prior to transfection, HepG2 cells were seeded in 6-well plates at a concentration of $2.5 \times 10^{5} /$ well, until $50-70 \%$ confluency was reached at the time of transfection. Transfection efficiency was analyzed by flow cytometry (Beckman Coulter, Miami, FL, USA). In this experiment, the optimal siRNA concentration for transfection was $100 \mathrm{nmol} / \mathrm{l}$. At 24, 36, 48 and $72 \mathrm{~h}$ following transfection, cells were processed and analyzed as described in each experiment.

Reverse transcription-PSCR (RT-PCR). Cells were collected $36 \mathrm{~h}$ following siRNA transfection and total RNA was
Table I. siRNA sequences for RNA interference.

\begin{tabular}{lc}
\hline siRNA & \multicolumn{1}{c}{ Nucleotide sequence of siRNA } \\
\hline Control & \\
Sense & 5'-UUCUCCGAACGUGUCACGUTT-3' \\
Antisense & 5'-ACGUGACACGUUCGGAGAATT-3' \\
siRNA-A & \\
Sense & 5'-GGGCUUUGAAUCAGCUAAATT-3' \\
Antisense & 5'-UUUAGCUGAUUCAAAGCCCTT-3' \\
siRNA-B & \\
Sense & 5'-GGCCCAGUUAAAGGAAACUTT-3' \\
Antisense & 5'-AGUUUCCUUUAACUGGGCCTT-3' \\
siRNA-C & \\
Sense & 5'-GAGGCAAGUAAGUCCAUAUTT-3' \\
Antisense & 5'-AUAUGGACUUACUUGCCUCTT-3'
\end{tabular}

Table II. Primer sequences for reverse transcription-PCR.

\begin{tabular}{lc}
\hline Primers & \multicolumn{1}{c}{ Nucleotide sequence of primers } \\
\hline SnoN & \\
Forward & 5'-AGAGACTCTGTTTGCCCCAAGT-3' \\
Reverse & 5'-CATGCTAAACTTCTCCTTCATTTC-3' \\
$\beta$-actin & \\
Forward & 5'-TTCTGTGGCATCCACGAAACT-3' \\
Reverse & 5'-GAAGCATTTGCGGTGGACGAT-3' \\
\hline
\end{tabular}

extracted using the RNA PCR kit (AMV) version 3.0 [Takara Biotechnology (Dalian) Co., Ltd.] as described by the manufacturer. For RT-PCR, $1 \mu \mathrm{g}$ total RNA was reverse transcribed in a $10 \mu \mathrm{l}$ reaction volume to form cDNA. The primer pairs for the PCR amplification of SnoN and $\beta$-actin were synthesized by Sangon Biotech (Shanghai) Co., Ltd. (China) and their sequences are listed in Table II. The PCR products of SnoN and $\beta$-actin were analyzed on $1.5 \%$ agarose gel, and the lengths for SnoN and $\beta$-actin were 584 and 312 bp, respectively. Total RNA of HepG2 cells was extracted with TRIzol reagent (Invitrogen Life Technologies). The ImProm-II Reverse Transcription (Promega Corporation, Madison, WI, USA) with $2 \mu \mathrm{g}$ of RNA and poly-dT primers was used for synthesis of cDNA. The amplified products were visualized on $1.5 \%$ agarose gels and then under UV light.

Western blot analysis. Cells were collected using $0.25 \%$ trypisn $72 \mathrm{~h}$ following siRNA transfection. To prepare the cell lysate, cells were incubated on ice for $15 \mathrm{~min}$ in cell lysis buffer (20 mM HEPES, pH 7.4; $150 \mathrm{mM} \mathrm{NaCl} ; 12.5 \mathrm{mM}$ $\beta$-glycerophosphate; $1.5 \mathrm{mM} \mathrm{MgCl} ; 2 \mathrm{mM}$ EDTA; $10 \mathrm{mM}$ $\mathrm{NaF} ; 2 \mathrm{mM}$ DTT; $1 \mathrm{mM} \mathrm{Na} \mathrm{VO}_{4} ; 1 \mathrm{mM}$ PMSF; $100 \mathrm{U} / \mathrm{ml}$ aprotinin and $0.5 \%$ Triton $\mathrm{X}-100)$. Total protein concentration was measured and $20 \mu \mathrm{g}$ protein were resolved on $10 \%$ SDS-PAGE gel. Subsequently, proteins were transferred to a polyvinylidene difluoride membrane (PVDF). Following 
blocking with 5\% non-fat milk for $1 \mathrm{~h}$, the membrane was probed consecutively with primary and peroxidase-conjugated secondary antibodies, and the signal was detected. The primary and secondary antibodies used in this study are as described previously. The dilution ratio for each antibody was 1:3,000.

Cell proliferation analysis by $C C K-8$. Cells were collected $24 \mathrm{~h}$ following siRNA transfection and re-plated at the same density per well into 96-well plates. Following incubation for $0,24,48,72$ and $96 \mathrm{~h}$ (1-5 days after siRNA transfection, respectively), cell proliferation was measured using the CCK- 8 kit. Briefly, $10 \mu$ l CCK-8 was added to each well and incubated for $1.5 \mathrm{~h}$. The OD value at $405 \mathrm{~nm}$ was measured.

Cell apoptosis analysis by annexin V/PI Kit. Cultured cells were trypsinized using $0.25 \%$ trypsin without EDTA $48 \mathrm{~h}$ following siRNA transfection, and washed once with cold PBS. According to the manufacturer's protocol, cells were resuspended in $1 \mathrm{X}$ annexin-binding buffer, and stained with Alexa Fluor ${ }^{\circledR} 488$ annexin V and PI (Invitrogen Life Technologies) for $15 \mathrm{~min}$ at room temperature prior to flow cytometric analysis.

Statistical analysis. SPSS software was used for statistical analysis and the experimental data were expressed as the mean \pm standard deviation. Single factor analysis of variance and the two-sample Student's t-test were performed to determine the statistical significance of the differences. $\mathrm{P}<0.05$ was considered to indicate a statistically significant difference.

\section{Results}

Effects of siRNA on the expression level of SnoN mRNA. To examine the effect of siRNAs on SonN gene expression, HepG2 cells were transfected with siRNA-A, -B and -C. Cells were also treated with a negative control siRNA and a blank control. The expression level of SnoN mRNA was analyzed by RT-PCR $36 \mathrm{~h}$ following siRNA transfection. The RT-PCR results are shown in Fig. 1. Following normalization to the gray value of the $\beta$-actin fragment, the gray value of the SnoN fragment of the blank control, negative control, and siRNA-A, -B and $-C$ groups were $0.655 \pm 0.105,0.591 \pm 0.039,0.251 \pm 0.056$, $0.469 \pm 0.063$ and $0.222 \pm 0.047$, respectively. Statistically, the expression level of SnoN mRNA in the siRNA-A, -B and $-\mathrm{C}$ groups was significantly reduced compared with the two control groups $(\mathrm{P}<0.05)$. Of the three siRNA fragments (siRNA-A, -B and -C), siRNA-C demonstrated the highest inhibition efficiency, with the expression level of SnoN mRNA decreasing by $62.4 \%$ compared with the negative control group. No significant difference was observed between the blank control and negative control groups.

Effects of siRNA on the expression level of SnoN protein. To verify the RT-PCR result, we analyzed the expression level of SnoN protein by western blot analysis following $72 \mathrm{~h}$ of siRNA transfection (Fig. 2). Following normalization to the gray value of $\beta$-actin, the gray values of the SnoN protein of the blank control, negative control, and siRNA-A, -B and-C groups were $0.832 \pm 0.049,0.786 \pm 0.043,0.463 \pm 0.026,0.682 \pm 0.034$ and $0.432 \pm 0.010$, respectively. Similar to the RT-PCR result,

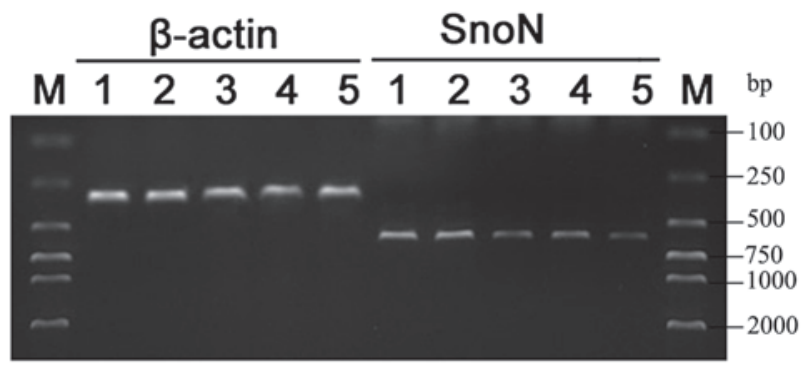

Figure 1. Reverse transcription PCR (RT-PCR) analysis of SnoN expression at the mRNA level. Cells were processed for RT-PCR analysis $36 \mathrm{~h}$ following siRNA transfection. The PCR products of SnoN and $\beta$-actin were analyzed on $1.5 \%$ agarose gel, and the length for SnoN and $\beta$-actin was 584 and 312 bp, respectively. $\beta$-actin was used as an internal control. The gray value of each lane represents the mRNA expression level of SnoN and $\beta$-actin. M, marker; 1, blank control; 2 , negative control; 3, siRNA-A; 4, siRNA-B; 5, siRNA-C.

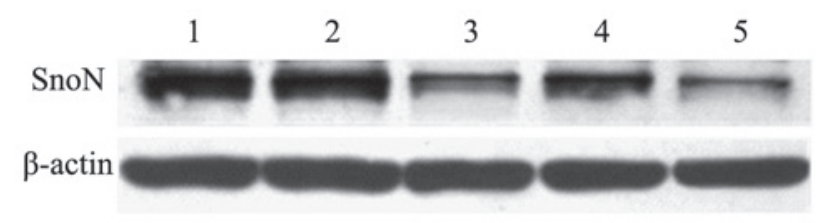

Figure 2. Western blot analysis of SnoN expression at the protein level Cells were collected and lysated for western blot analysis $72 \mathrm{~h}$ after siRNA transfection. $\beta$-actin was used as an internal control. Lanes: 1 , blank control; 2, negative control; 3, siRNA-A; 4, siRNA-B; 5, siRNA-C.

the expression level of SnoN protein in the siRNA groups was decreased compared with the two control groups. Statistically, there was a significant difference in expression between the siRNA-C group and the negative control group $(\mathrm{P}<0.01)$. There was no significant difference in expression between the blank control and the negative control groups. These results indicate that siRNA-C was able to efficiently inhibit SnoN gene expression, both at the mRNA and protein level. Therefore, siRNA-C was used as the interference fragment in the subsequent experiments.

Effect of SnoN downregulation on cell proliferation. It has been demonstrated that $\mathrm{SnoN}$ is important in regulating cell proliferation. Thus, we assessed the role of SnoN in HepG2 cell proliferation. Following efficient inhibition of the expression of SnoN by siRNA-C, the growth status of HepG2 cells was detected with CCK- 8 . The OD value at $405 \mathrm{~nm}$ is directly proportional to the number of living cells. As shown in Table III, on Days 1-5 of transfection, the OD value of the siRNA group was significantly lower than that of the blank control and the negative control groups. On Day 3, the cell viability of the HepG2 cells was the lowest with the OD value approximately half that of the blank control and the negative control groups, while on Day 4, cell viability began to recover. This result suggests that downregulation of SnoN reduced the cell viability of the HepG2 cells.

Effect of SnoN downregulation on apoptosis. SnoN is also important in regulating cell apoptosis. However, its involvement in HepG2 apoptosis remains unknown. In the present study, the cell apoptosis status was analyzed following $48 \mathrm{~h}$ 
Table III. The $\mathrm{OD}_{405}$ value of the blank group, the negative control group and the siRNA group.

$\mathrm{OD}_{405}$ value following transfection

\begin{tabular}{lccccc}
\cline { 2 - 5 } Group & \multicolumn{1}{c}{ Day 1 } & \multicolumn{1}{c}{ Day 2 } & Day 3 & Day 4 & Day 5 \\
\hline The blank control & $0.371 \pm 0.013$ & $0.807 \pm 0.036$ & $1.548 \pm 0.074$ & $2.024 \pm 0.109$ & $2.415 \pm 0.087$ \\
The negative control & $0.319 \pm 0.029$ & $0.636 \pm 0.041$ & $1.378 \pm 0.045$ & $1.858 \pm 0.096$ & $2.430 \pm 0.112$ \\
The siRNA group $^{\mathrm{a}, \mathrm{b}}$ & $0.258 \pm 0.033^{\mathrm{ab}}$ & $0.468 \pm 0.031^{\mathrm{a}, \mathrm{b}}$ & $0.687 \pm 0.023^{\mathrm{a}, \mathrm{b}}$ & $1.064 \pm 0.036^{\mathrm{a}, \mathrm{b}}$ & $1.854 \pm 0.088^{\mathrm{a}, \mathrm{b}}$ \\
\hline
\end{tabular}

${ }^{\text {a }}<0.05$ siRNA group vs. negative control group; ${ }^{\mathrm{b}} \mathrm{P}<0.01 \mathrm{siRNA}$ group vs. blank group. Mean \pm standard deviation, $\mathrm{n}=3$.

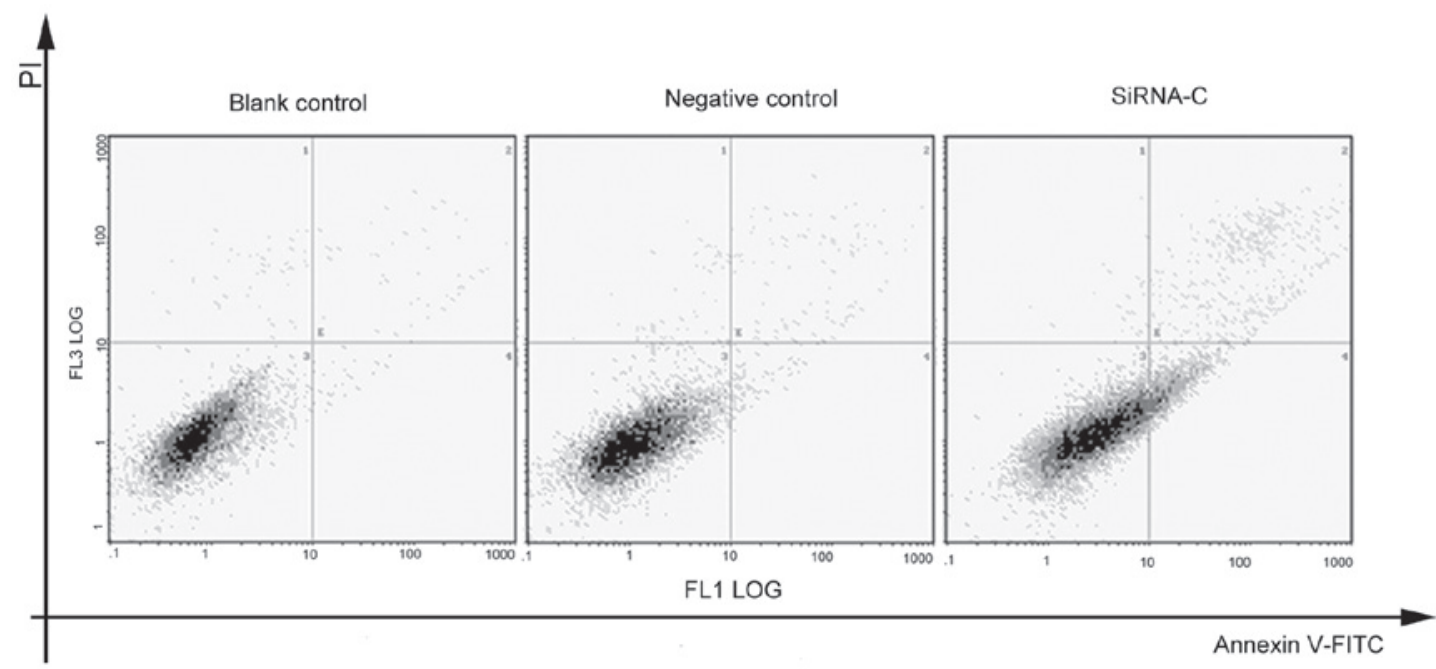

Figure 3. Assessment of apoptosis by flow cytometry using annexin V labeling. Cells were collected and labeled with annexin V and PI $48 \mathrm{~h}$ following siRNA-C transfection. Experiments were performed three times and representative analyses are shown.

of siRNA transfection. Fig. 3 shows the representative results of apoptosis, as analyzed by flow cytometry. The percentage of apoptotic cells in the siRNA-C group (9.84 $\pm 2.28 \%$ ) was significantly higher than that of the blank control group $(0.65 \pm 0.56 \%)$ and the negative control group $(1.78 \pm 1.25 \%)$ $(\mathrm{P}<0.05)$. These data indicate that induction of apoptosis in HepG2 cells was increased following downregulation of SnoN.

\section{Discussion}

RNAi technology is a powerful research tool that is able to induce gene silencing at the mRNA level. It selectively and directly targets a specific sequence of mRNA, rendering it an ideal research tool to study gene function (18). In RNAi technology, it is convenient and economical to used synthesized siRNA duplexes that are capable of interfering with gene expression with rapid, specific and robust effects. In this study, we knocked down SnoN gene expression by using siRNA silencing in HepG2 cells, and studied its role in liver cell proliferation and apoptosis. Our results demonstrated that all three siRNAs (siRNA-A, -B and -C) were effective at inhibiting SnoN expression at both the mRNA (Fig. 1) and protein (Fig. 2) level. The optimal inhibition efficiency was $62.4 \%$ and was by siRNA-C. Therefore, siRNA-C was used for the inhibition of SnoN expression in the cell proliferation and apoptosis analysis. The interfering effect of siRNA partly depends on the second structure of the target sequence. The three siRNAs (siRNA-A, -B and -C) used in our study targeted different sequences of SnoN and had varying interfering effects. Thus, the design of more effective siRNA sequences requires further investigation.

Cell proliferation was detected by CCK-8, which allows for sensitive colorimetric assays for the determination of cell viability in cell proliferation assays. In this kit, a highly water-soluble tetrazolium salt is reduced by the dehydrogenase activities in cells to give a yellow formazan dye, which is soluble in the tissue culture media. The quantity of formazan dye, generated by the activities of the dehydrogenases in the cells, is directly proportional to the number of living cells. The detection sensitivity of CCK-8 is higher than that of the other tetrazolium salts such as MTT. In addition, it is non-toxic to cells and allows for long incubation of cells. The results revealed that HepG2 cell viability was decreased following blocking of SnoN expression by siRNA-C. On the third day of transfection, cell viability was the lowest; while on the fourth day, cell viability began to recover. This may be caused by the transient effect following transient transfection (3-5d) of chemically synthesized siRNA. Notably, these data are consistent with those of Bravou et al and Band et al $(11,19)$ on colorectal and breast cancer. Our results suggest that SnoN is 
capable of promoting liver cell proliferation and has a role in liver tumorigenesis.

Annexin V/PI is an effective and sensitive way to analyze cell apoptosis. In our study, apoptosis in cells with inhibited SnoN expression was significantly higher. These data suggest that SnoN plays a role in the inhibition of HepG2 cell apoptosis. In summary, the present study has demonstrated that the SnoN gene acts as an oncogene in HepG2 cells, and is important in the promotion of liver cancer cell proliferation and the inhibition of apoptosis. The underlying mechanisms of these findings, and whether they involve the TGF- $\beta /$ Smad signaling pathway, require further investigation.

\section{References}

1. Deheuninck J, Luo K: Ski and SnoN, potent negative regulators of TGF-beta signaling. Cell Res 19: 47-57, 2009.

2. Jahchan NS and Luo K: SnoN in mammalian development, function and diseases. Curr Opin Pharmacol 10: 670-675, 2012.

3. Bonni S and Bonni A: SnoN signaling in proliferating cells and postmitotic neurons. FEBS Lett 586: 1977-1983, 2012

4. Stroschein SL, Wang W, Zhou S, et al: Negative feedback regulation of TGF- $\beta$ signaling by the SnoN oncoprotein. Science 286 : 771-774, 1999.

5. Pan D, Zhu Q, Conboy MJ, Conboy IM and Luo K: SnoN activates p53 directly to regulate aging and tumorigenesis. Aging Cell 11: 902-911, 2012.

6. Javelaud D, van Kempen L, Alexaki VI, et al: Efficient TGF- $\beta$ / SMAD signaling in human melanoma cells associated with high c-SKI/SnoN expression. Mol Cancer 10: 2-14, 2011.

7. Pan D, Zhu Q and Luo K: SnoN functions as a tumour suppressor by inducing premature senescence. EMBO J 28: 3500-3513, 2009.
8. Tecalco-Cruz AC, Sosa-Garrocho M, Vázquez-Victorio G, et al: Transforming growth factor $\beta$-/SMAD target gene SKIL is negatively regulated by the transcriptional cofactor complex SnoN-Smad4. J Biol Chem 287: 26764-26776, 2012.

9. Krakowski AR, Laboureau J, Mauviel A, et al: Cytoplasmic SnoN in normal tissues and nonmalignant cells antagonizes TGF-beta signaling by sequestration of the Smad proteins. Proc Natl Acad Sci USA 102: 12437-12442, 2005.

10. Zhu Q, Krakowski A R, Dunham E E, et al: Dual role of SnoN in mammalian tumorigenesis. Mol Cell Biol 27: 324-339, 2007.

11. Bravou V, Antonacopoulou A, Papadaki H, et al: TGF-beta repressors SnoN and Ski are implicated in human colorectal carcinogenesis. Cell Oncol 31: 41-51, 2009.

12. Shinagawa T, Dong HD, Xu M, Maekawa T and Ishii S: The sno gene, which encodes a component of the histone deacetylase complex, acts as a tumor suppressor in mice. EMBO J 19: 2280-2291, 2000.

13. Jahchan NS, Wang D, Bissell MJ and Luo K: SnoN regulates mammary gland alveologenesis and onset of lactation by promoting prolactin/Stat5 signaling. Development 139: 3147-3156, 2012.

14. Jahchan NS, You YH, Muller WJ and Luo K: Transforming growth factor-beta regulator SnoN modulates mammary gland branching morphogenesis, postlactational involution, and mammary tumorigenesis. Cancer Res 70: 4204-4213, 2010.

15. Akagi I, Miyashita M, Makino H, et al: $\mathrm{SnoN}$ overexpression is predictive of poor survival in patients with esophageal squamous cell carcinoma. Ann Surg Oncol 15: 2965-2975, 2008.

16. Chang K, Marran K, Valentine A and Hannon GJ: RNAi in cultured mammalian cells using synthetic siRNAs. Cold Spring Harb Protoc 2012: 956-961, 2012.

17. Hannon GJ: RNA interference. Nature 418: 244-251, 2002.

18. Conde J, Ambrosone A, Sanz V, et al: Design of multifunctional gold nanoparticles for in vitro and in vivo gene silencing. ACS Nano 6: 8316-8324, 2012.

19. Band AM and Laiho M: SnoN oncoprotein enhances estrogen receptor- $\alpha$ transcriptional activity. Cell Signal 24: 922-930, 2012 . 